\title{
Photo-crosslinkable, deformable PMMA colloids
}

\begin{abstract}
Matthias K. Klein, ${ }^{a}$ Andreas Zumbusch ${ }^{\star a}$ and Patrick Pfleiderer ${ }^{\mathrm{b}}$
Polymeric nano and microparticles are finding widespread applications in materials and biological science. In all preparation methods pursued to date, the chemical stability of the particles is determined by the polymer system chosen. Usually, after particle synthesis, postprocessing with the aim of changing their stability is not possible. Therefore, one only has two choices: the synthesis of crosslinked polymer particles with high solvent and heat resistance and limited formability, or the synthesis of polymer particles consisting of linear polymer chains exhibiting low stability and high formability. In this contribution, we describe a new class of colloids which combine the advantages of both particle systems. The particles are composed of linear PMMA chains which are functionalized with photo crosslinkable moieties. After preparation, the particles can be deformed before they are crosslinked by UV illumination. This offers unique possibilities for particle modification with a broad range of possible applications. Three examples are given. First, we show that the particles can be thermo mechanically deformed into anisotropic shapes, in which they can be locked by subsequent crosslinking. Relaxation into the thermodynamically favorable spherical shape is thus suppressed, even in harsh environments. Second, we describe how the difference in solvent stability between illuminated and non illuminated particles can be exploited for photolithography. Third, we demonstrate how photo crosslinked anisotropic particles can be loaded with functional compounds, e.g. fluorophores.
\end{abstract}

\section{Introduction}

Polymeric nano- and microparticles represent an important class of materials. As dispersions they find widespread practical use, for example in paints and coatings. At the same time, they are of equal importance in basic and applied research, e.g. as colloidal model systems ${ }^{14}$ or for the formation of photonic crystal materials. ${ }^{5}$ In general, the stability of the particles is determined by the chosen polymer system. Thus, in general factors like solvent stability or thermo-mechanical properties cannot be changed after the particle synthesis step. This, however, severely limits the range of possible applications of these systems.

Particles composed of poly(methyl methacrylate) (PMMA) have proven to be a versatile colloidal system. Like other polymer particles, PMMA particles can be composed of either linear or crosslinked polymer chains. ${ }^{8}$ In the first case, the particles can be thermo-mechanically deformed but show limited solvent resistance and low thermal shape stability. By contrast, particles composed of crosslinked PMMA are very stable but permanent

${ }^{a}$ Department of Chemistry, University of Konstanz, 78457 Konstanz, Germany. E mail: andreas.zumbusch@uni konstanz.de; Fax: +49 753188 3139; Tel: +49 7531882357 ${ }^{b}$ Department of Physics, University of Konstanz, 78457 Konstanz, Germany. E mail: patrick.pfleiderer@uni konstanz.de; Fax: +49 (0) 7531 88 3090; Tel: +49 (0) 7531 882014 deformation is precluded. In general, PMMA particles can be prepared using a dispersion polymerization procedure in nonaqueous media first reported by Osmond and Thompson. ${ }^{9}{ }^{12}$

Over the decades, many variants of this basic preparation procedure have been published. For example, the preparation of different kinds of fluorescently labeled PMMA model colloids, which are suitable for confocal fluorescence microscopic studies, has been described. ${ }^{2,13}{ }^{16}$ After their preparation, all of these particles have a spherical shape. Rich additional dynamics are, however, expected for non-spherical systems. Monodisperse shape-anisotropic microparticles have therefore recently met a lot of interest. ${ }^{17}{ }^{27} \mathrm{~A}$ well-established method for the generation of elliptical polymer particles is the stretching procedure pioneered by Keller and coworkers. ${ }^{28}$ For this purpose, the polymer particles are embedded in crosslinked polymer films, which are subsequently heated well above the particle glass transition temperature and finally stretched. This leads to elliptical particles with strain-defined aspect ratios. On this basis, Solomon and coworkers have prepared sterically stabilized, fluorescent PMMA model colloids of elliptical shape. ${ }^{17}$ Steric stabilization is the method of choice when seeking hard-core interactions among the particles. ${ }^{29}$ Furthermore, controlled degradation of the stretching matrix can be used to produce PMMA ellipsoids with sticky ends. These can be stained with a fluorescent steric stabilizer as has been demonstrated by Zhang et al. ${ }^{30}$ Starting from spherical PMMA microparticles, it is thus possible to generate ellipsoids and more complicated forms in a well-controlled manner. ${ }^{31}$ 
However, since these particles are composed of linear PMMA chains to allow for thermomechanic deformation, they tend to reduce surface energy and relax back to the thermodynamically favored spherical shape in many solvents or at elevated temperatures. The only possibility to lock the particles in their anisotropic shape is the subsequent generation of a polymer network in the particles. This can be achieved by interconnecting the properly functionalized polymer chains by a photo-chemical reaction right after the deformation process as it has been developed for many polymer systems. ${ }^{32} 37$

Inspired by this, we have prepared micron-sized sterically stabilized PMMA colloids composed of a PMMA copolymer bearing a photo-reactive functionality. ${ }^{38,39}$ Well-known molecular functionalities which undergo photo-dimerization during illumination with UV light are cinnamic acid and many of its derivatives. ${ }^{39}{ }^{44}$ We covalently bound such functional groups to the PMMA backbones. UV illumination then interconnects the polymer chains in each particle by truxillate units, which are generated via the photo-induced $[2 \pi+2 \pi]$ cycloadditon of two neighbouring cinnamoyl residues (see Scheme 1).

In order to introduce the photo-reactive functionality, we selected the commercial photo-crosslinking comonomer cinnamoyl oxyethyl acrylate (CEA), which is well established in photolithographic applications and was already used for the fabrication of light sensitive shape memory polymers. ${ }^{42,45}$

The preparation of polymer particles consisting of linear PMMA bearing photo-crosslinkable moieties opens a broad range of possible applications. We describe three examples in which the particles are engineered after the preparation step. First, we demonstrate that large quantities of thermally deformed colloids can be locked in an anisotropic shape by illumination with UV

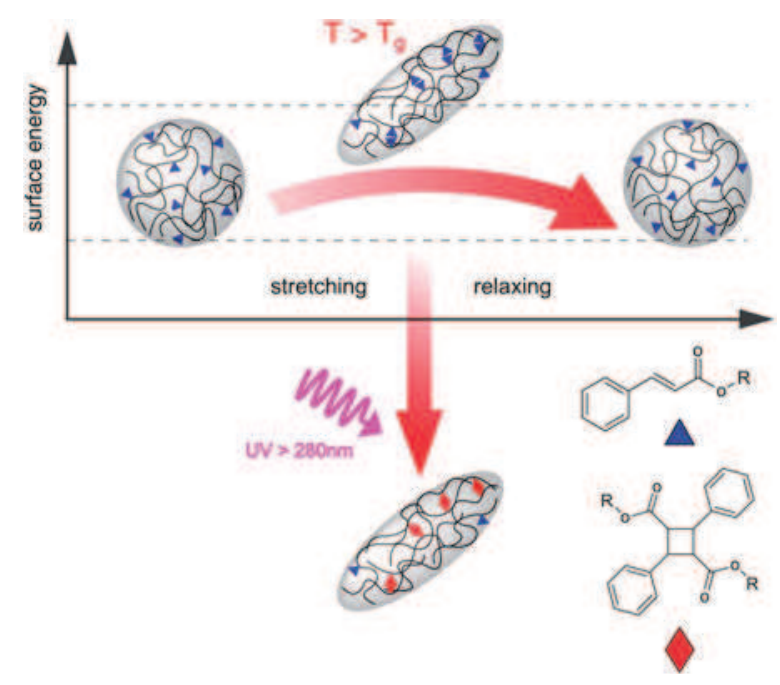

Scheme 1 Generation of formstable elliptical PMMA particles. Photo cross linkable spherical PMMA particles are obtained by copolymerizing MMA with CEA resulting in polymer chains $(R)$ bearing cinnamoyl residues (blue triangles) Stretching at elevated temperatures $\left(T>T_{\mathrm{g}}\right)$ without subsequent illumination leads to ellipsoids. At temperatures above $T_{\mathrm{g}}$ or in the presence of certain solvents the ellipsoids relax back to the spherical shape in order to reduce surface energy. This relaxation behaviour is observed for common PMMA ellipsoids as well. The formation of truxillate connections (red diamonds) caused by UV illumination leads to locking of the elliptical shape. light. Second, we show how the patterned illumination of ordered particle layers can be used to generate macroscale colloidal crystalline patterns. This photolithographic patterning and the shape locking rely on the substantially increased thermal stability and solvent resistance of the colloids after UV illumination. Finally, as a third application example we depict how the dense polymer network of the photo-crosslinked, shape-anisotropic particles can be exploited to load them with nano-sized, functional compounds by a simple swelling/deswelling procedure, ${ }^{13}$ in our case with a fluorescent dye.

\section{Experimental section}

\section{Materials and methods}

Methyl methacrylate (MMA), methacrylic acid (MA), octanethiol (OctSH), azobisisobutyronitrile (AIBN), dodecane, hexane, polydimethylsiloxane $\left(M_{\mathrm{n}} \sim 110000\right.$; viscosity $\left.\sim 50000 \mathrm{cSt}\right)$ (PDMS), methylhydro-dimethyl siloxane copolymer $\left(M_{\mathrm{n}} \sim 950\right.$, methylhydroxysiloxane $50 \mathrm{~mol} \%$ ), stannous 2-ethylhexanoate, decahydronaphthalene (decalin) (mixture of cis + trans isomers, reagent grade 98\%), butyl acetate (BA) and ethyl acetate (EA) were purchased from Sigma-Aldrich. The photo-crosslinking comonomer 2-cinnamoyl oxyethylacrylate (CEA) was supplied by Polyscience Europe. Before particle preparation MMA was purified over basic aluminium oxide (Woelm Pharma) and methacrylic acid was distilled at reduced pressure.

UV/VIS spectra of spin cast polymer films were measured on a Cary 50 spectrometer (Varian). FT-IR spectra of dried particle samples were recorded on the ATR unit of a Spectrum 100 FT-IR spectrometer (Perkin Elmer). Gel Permeation Chromatography (GPC) was performed on a Polymer Laboratories PL 50 instrument equipped with two PLgel $5 \mu \mathrm{m}$ MIXED-C columns in THF with both a RI- and an UV-detector. ${ }^{1} \mathrm{H}$-NMR spectra were recorded with a Bruker Avance III-400 (400 MHz) spectrometer. Particle stretching was conducted using a homebuilt stretching apparatus driven by a stepper motor. Scanning Electron Microscopy (SEM) was performed on a CrossBeam 1540XB (Zeiss) at an acceleration voltage of $1 \mathrm{kV}$. Samples were dried on silicon wafers and sputter coated with $\sim 5 \mathrm{~nm}$ gold layers in order to enhance conductivity. TEM images were recorded on a Libra 120 instrument at an acceleration voltage of $120 \mathrm{kV}$. Differential Interference Contrast (DIC) images were recorded on a Leica DMI 6000B microscope using a $100 \times / 1.4-0.7$ oilimmersion CS objective (Leica). Confocal fluorescence imaging was performed on a Leica LSM laser scanning microscope using a $100 \times$ oil immersion objective. Fluorescence was excited at $514 \mathrm{~nm}$ with an argon laser and fluorescence was detected between 550 and $600 \mathrm{~nm}$.

\section{Particle preparation using non-aqueous dispersion polymerization}

The reagents were weighed into a $50 \mathrm{ml}$ round bottom flask equipped with a magnetic stir bar and a reflux condenser. (Details on the composition of the polymerization mixture are given in Table S1.†) After the AIBN was completely dissolved, the flask was deepened into an oil bath which was preheated to 
$95{ }^{\circ} \mathrm{C}$. The stirring speed was set to $250 \mathrm{rpm}$. After approximately $3 \mathrm{~min}$ the reagent mixture became turbid indicating particle nucleation. The reagent mixtures were stirred for an additional $2.5 \mathrm{~h}$ at $95^{\circ} \mathrm{C}$. The final particle dispersions LPX01, LPX02, LPX03 and LPX04 were filtered over glass wool. Each particle batch was centrifuged and washed 3 times with hexane.

\section{Particle stretching}

$2.4 \mathrm{~g}$ of the dried particles LPX02 dispersed in $59.4 \mathrm{~g}$ hexane was mixed with $224.2 \mathrm{~g}$ of a $33.9 \%$ (w/w) PDMS/hexane solution. After the addition of $0.29 \mathrm{~g}$ of the crosslinker methylhydrodimethyl siloxane copolymer and $0.34 \mathrm{~g}$ of the catalyst stannous 2-ethylhexanoate, each dissolved in $12 \mathrm{ml}$ of hexane, the dispersion was filled into a PTFE coated mould. Most of the hexane was allowed to evaporate during $15 \mathrm{~h}$ and the PDMS film was completely crosslinked at $125{ }^{\circ} \mathrm{C}$ during $5 \mathrm{~h}$. The resulting film was cut into $2 \mathrm{~cm}$ wide strips which were fixed between the two movable clamps of the mechanical stretching apparatus. The strips were heated in air to $165{ }^{\circ} \mathrm{C}$ and continuously stretched by a factor of 2.3 over one hour. After cooling to room temperature, the film was cut into $2 \mathrm{~cm} \times 2 \mathrm{~cm}$ pieces which were subsequently swollen in hexane for $16 \mathrm{~h}$ and finally degraded in a solution of sodium methanolate and isopropanol in hexane to release the stretched particles. The degrading mix was composed of $3.47 \%$ PDMS film, $78.81 \%$ hexane, $17.72 \%$ isopropanol and $0.04 \%$ sodium methoxide by weight. In order to remove film fragments and degrading agents the dispersion was centrifuged at $4000 \mathrm{rpm}$ for $10 \mathrm{~min}$ and washed with hexane/isopropanol $(75: 25(\mathrm{v} / \mathrm{v}))$. After the third repetition the supernatant was discarded and replaced by $10 \mathrm{ml}$ of decalin and the particles were restabilized by adding $2 \mathrm{ml}$ of a solution containing $5 \%(\mathrm{w} / \mathrm{w})$ of the steric stabilizer dissolved in a mixture of BA/EA $(1: 2(\mathrm{w} / \mathrm{w}))$. The ellipsoids were sonicated for 15 min to ensure adsorption of the steric stabilizer. Subsequently the particles were washed 2 times with decalin and finally dispersed in $10 \mathrm{ml}$ of decalin to yield the final dispersion.

\section{Particle illumination}

$2 \mathrm{ml}$ of the respective particle dispersions in decalin was irradiated at ambient temperature $\left(22^{\circ} \mathrm{C}\right)$ in a quartz cuvette sealed with a PTFE stopper at particle concentrations between $17.5 \mathrm{mg}$ $\mathrm{ml}^{-1}$ and $35 \mathrm{mg} \mathrm{ml}^{-1}$. The dispersions were agitated using a magnetic stir bar to prevent particle settling. Irradiation was performed by focusing the light of a $200 \mathrm{~W}$ high pressure mercury short arc lamp (HBO $200 \mathrm{~W} / 2 \mathrm{~L} 1$; Osram) to the middle part of the cuvette. Heating of the sample was prevented by a water cooled cuvette holder and the IR irradiation was blocked by a quartz cell filled with aqueous copper sulphate solution placed in the light path. Deep UV light with wavelengths $<280 \mathrm{~nm}$ was blocked by a glass filter.

\section{Results and discussion}

\section{Preparation of photo-crosslinkable PMMA particles}

We prepared the photo-crosslinkable PMMA precursor particles by the well established non-aqueous dispersion copolymerization of methyl methacrylate (MMA), methacrylic acid (MA), and CEA with varying molar ratios between MMA and CEA in an aliphatic hydrocarbon mixture. Our investigations revealed no significant influence of the selected comonomer contents on the polydispersity of the resulting particle size distributions. The different particle samples show low polydispersities which is reflected in the extended colloidal crystalline areas which are formed upon drying of diluted dispersions in hexane. Classic chemical crosslinkers such as ethylene glycol dimethacrylate (EGDMA) can seriously disturb the particle nucleation process resulting in rather broad size distributions. ${ }^{\mathbf{8} 46,47}$ Therefore, common crosslinking reagents like EGDMA are usually added right after particle nucleation in order to prevent the disturbance at that crucial step. In our case, a delayed addition of the photo-crosslinking comonomer can be eluded, simplifying the polymerization process. In addition, the desired particle diameter can be predetermined by the variation of the initial monomer mixture/dispersant weight ratio as discussed in the SI. This has already been described by Antl et al. for common PMMA model colloids. ${ }^{10,15}$ Using this modified polymerization procedure, we synthesized photo-crosslinkable PMMA colloids with diameters ranging from $1.15 \mu \mathrm{m}$ to $1.81 \mu \mathrm{m}$ (Fig. S1†). The current size range can easily be extended to smaller particle diameters, down to a few hundreds of nanometers. ${ }^{10}$ For all particle batches reported herein the polydispersities were below $4 \%$. Individual particle distributions were extracted from Scanning Electron Microscopy (SEM) images. ${ }^{1} \mathrm{H}-\mathrm{NMR}$ experiments indicated that the photo-crosslinking comonomer CEA was almost completely incorporated into the final polymer particles (see ESI $\dagger$ ). In order to quantify the amount of incorporated cinnamoyl residues in the polymer chains we compared the integrals of the methoxy protons of the polymer backbone to the resonances corresponding to the vinyl and aromatic protons of the cinnamoyl residues (see ESI $\dagger$ ). In addition gel permeation chromatography (GPC) revealed the statistical character of the resulting copolymer (Fig. S2 $\uparrow$ ). Size distributions recorded by UV- and refractive index (RI)-detection were almost identical. This demonstrates that the cinnamoyl groups, which can be detected by their strong absorption located at $280 \mathrm{~nm}$, are randomly distributed over all polymer chains, which leads to the congruent distribution plots depicted in Fig. S2. $\dagger$ We set a molar ratio of $2.7 \%(\mathrm{n} / \mathrm{n})$ of the comonomer as an upper limit for the photo-crosslinking, since up to this monomer ratio, the unwanted gel content prior to illumination was negligible. We have also investigated higher CEA contents up to $5.4 \%$ and determined gel contents up to $79 \%$ right after polymerization. Their high degree of crosslinking makes these particles inapplicable for the thermomechanical stretching experiments described in the following.

\section{Photo-crosslinking of PMMA microparticles}

In the following we report the photo-crosslinking behavior of the spherical precursor particles. In order to guarantee homogeneous crosslinking of the particles we decided to perform the illumination procedure on freely diffusing particles in dispersion. The random diffusion of the colloids through the illuminated volume leads to similar illumination doses for each particle. In addition this procedure is suited for the 
illumination of a large amount of particles at once. Typically, we have illuminated between $35 \mathrm{mg}$ and $70 \mathrm{mg}$ of dried particles in one run. We performed the illumination procedure at ambient temperature, well below the $T_{\mathrm{g}}$ of PMMA. Under these conditions the PMMA chain mobility is drastically reduced and shape relaxation during photo-crosslinking, especially of thermomechanically deformed colloids, is suppressed. Prior to illumination we dispersed the particles in decalin because this dispersant is transparent above $280 \mathrm{~nm}$ and has a refractive index similar to that of the dispersed PMMA particles. The low refractive index contrast reduces scattering and makes light penetration into the freely diffusing colloids more efficient. In order to determine the appropriate illumination conditions, the spherical precursor particles were illuminated in dispersion for varying durations, washed with hexane, dried, and dissolved in dichloromethane. The resulting polymer solutions were then spin cast on quartz slides in order to prepare thin polymer films for investigation by UV-VIS spectroscopy. As illustrated in Fig. S5, $\uparrow$ longer illumination times lead to a decreasing absorption intensity of the cinnamic acid band. This can be attributed to the photo-induced formation of truxillate connections (red diamonds in Scheme 1) between the polymer chains. In addition, an increasing scattering background due to microgel formation in the spin cast polymer films is observed. Other than with UV-VIS spectroscopy, the photo-crosslinking process can also be monitored by Fourier Transform (FT)-IR spectroscopy. For the intermediate sized particles with a diameter of $1.6 \mu \mathrm{m}$, denoted as LPX02, after $6 \mathrm{~h}$ of illumination the photodimerization of the cinnamic acid residues resulted in a pronounced decrease of the IR absorption band located at $1637 \mathrm{~cm}^{-1}$. The latter is assigned to the absorption of the vinylene $\mathrm{C}=\mathrm{C}$ stretching vibration of the cinnamoyl groups (Fig. S6 $\dagger$ ), which decreases upon photodimerization. In order to quantify the conversion, we selected the $\mathrm{C}-\mathrm{CH}_{3}$ bending vibration located at $1388 \mathrm{~cm}^{-1}$ as an internal reference because the polymeric backbone is not affected by the photochemical reaction. The fraction $\mathrm{F}$ of photo-dimerized cinnamoyl groups can then be calculated according to ${ }^{45}$

$$
F=1 \quad \frac{\left(A_{1637} / A_{1388}\right)_{6 h}}{\left(A_{1637} / A_{1388}\right)_{0 h}}
$$

The numerator of the quotient is the ratio of the IR absorption of the illuminated particles which is divided by the absorption ratio before illumination. Regarding the respective absorption, the calculation leads to a value of $F=0.45$, meaning that $45 \%$ of the cinnamoyl residues have dimerized during illumination. Considering the number averaged molecular weight of $45000 \mathrm{~g} \mathrm{~mol}^{-1}$ determined by GPC, this corresponds to approximately 5 crosslinks per polymer chain, which is sufficient to form a dense polymer network in the particles. Moreover, we have investigated the gelation behavior during illumination for the three batches of differently sized particles (Fig. 1A). After $6 \mathrm{~h}$ of illumination, the photo-crosslinking led to gel contents between $84 \%$ and $88 \%$. The photo-induced gelation saturates at around $90 \%$ gel content after 7 hours of illumination for each particle batch. This means that after $6 \mathrm{~h}$ of illumination the majority of the polymer mass is forming a network and only $10 \%$ of the total polymer mass is not connected to the polymer network and thus can be extracted by the good solvents tetrahydrofuran (THF) or acetone.

Regarding the shape of the gelation curves, the gel-formation behavior has only a minor dependence on particle size in the studied size range. Furthermore, we followed the gelation of individual particles by SEM microscopy. The illuminated dispersions of LPX03 (white triangles in Fig. 1A) were diluted with a 100-fold excess of acetone. This leads to complete dissolution of linear, non-crosslinked PMMA. After sonicating
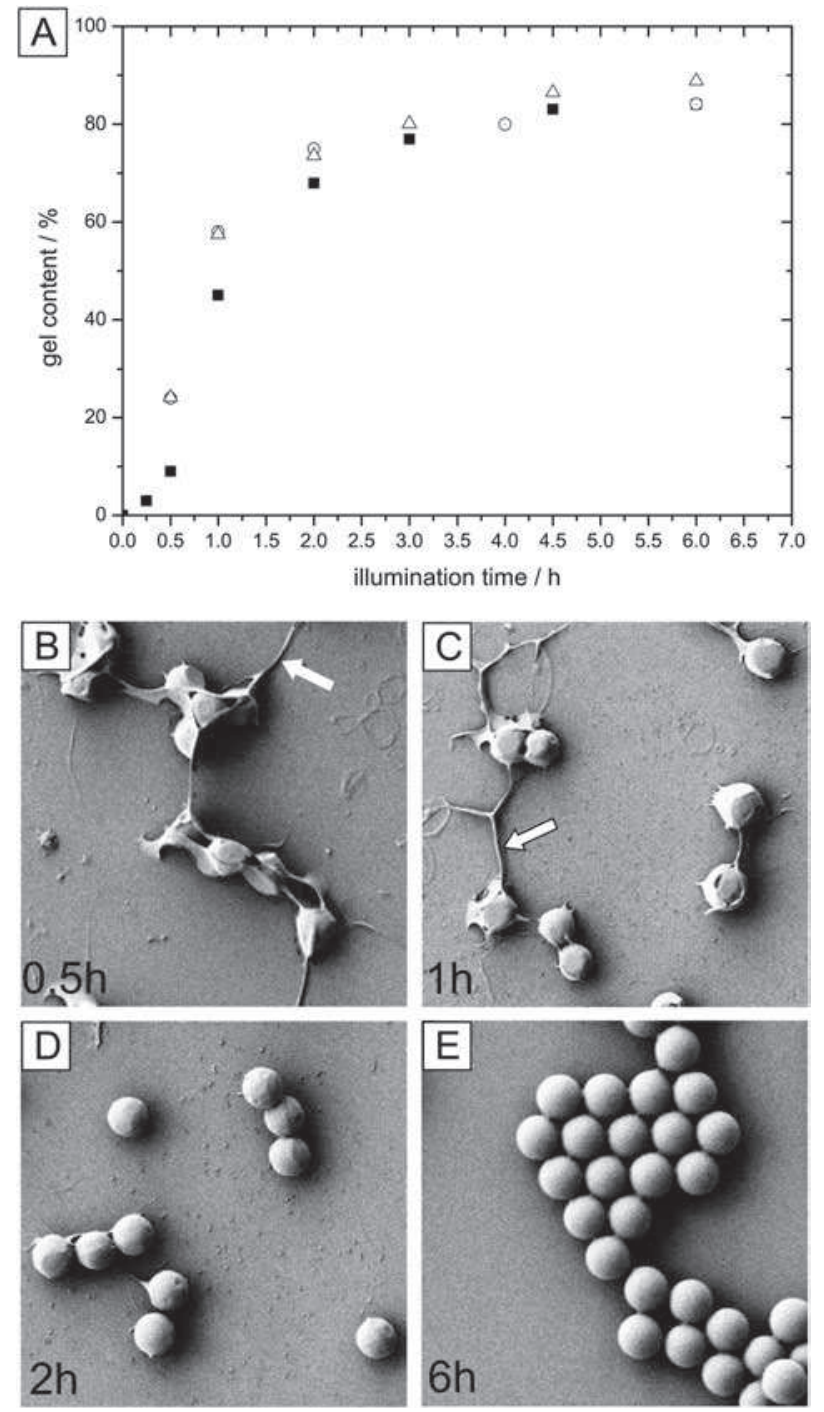

Fig. 1 (A) Gel content after different illumination times. The gravimetric gel content was determined by the solvent extraction method for the three differ ently sized, non fluorescent particles with mean diameters of $1.15 \mu \mathrm{m}$ (white circles), $1.6 \mu \mathrm{m}$ (black squares), and $1.81 \mu \mathrm{m}$ (white triangles) denoted as LPX01, LPX02, and LPX03 after different illumination times in dispersion. For each measurement $35 \mathrm{mg}$ of the respective particles was dispersed in $2 \mathrm{ml}$ of decalin. (B E) SEM images of acetone treated particles after different illumination times. Dispersions were mixed with a 100 fold excess of acetone and deposited on silicon substrates. Respective illumination times are indicated at the bottom left corner of each micrograph. The dimensions of the SEM micrographs are $15 \mu \mathrm{m} \times$ $15 \mu \mathrm{m}$ respectively. 
the samples for $10 \mathrm{~min}, 5 \mu \mathrm{l}$ of each dispersion was deposited on silicon wafers. After 30 min of illumination and subsequent dilution with acetone we could already observe single particles, which were however deformed and interconnected by extended fibers (Fig. 1B). These fibers (highlighted by white arrows in Fig. 1B and C) are composed of non-crosslinked PMMA, which was extracted by acetone. With increasing illumination times the particles better retain their structural integrity. After 2 hours of illumination (Fig. 1D), surface defects or any deformations after acetone treatment become rare and the particles remain intact. As illustrated in Fig. 1E, after $6 \mathrm{~h}$ of illumination the fiber-like structures between neighboring particles disappear. This is a direct indication of the high degree of crosslinking of the particles.

In order to investigate the thermal stability and the coalescence behavior of illuminated and non-illuminated particles well above the glass transition temperature of PMMA, twodimensional colloidal crystalline areas of illuminated and nonilluminated particles were deposited on microscopy coverslips and heated up to $200{ }^{\circ} \mathrm{C}$. The differential interference contrast (DIC) micrographs shown in Fig. 2 reveal that after $1 \mathrm{~h}$ the nonilluminated particles completely coalesce due to melting of the non-crosslinked polymer and the borderlines between adjacent particles are almost not observable anymore (Fig. 2b). By contrast, the photo-crosslinked particles only exhibit minor signs of coalescence (Fig. 2d). The micro-patterning of colloidal crystalline areas leads to Bragg diffraction at the macro-scale which completely disappears during tempering in the case of particle coalescence. ${ }^{48}$ In the case of the illuminated particles we explain the partial coalescence by the remaining mass

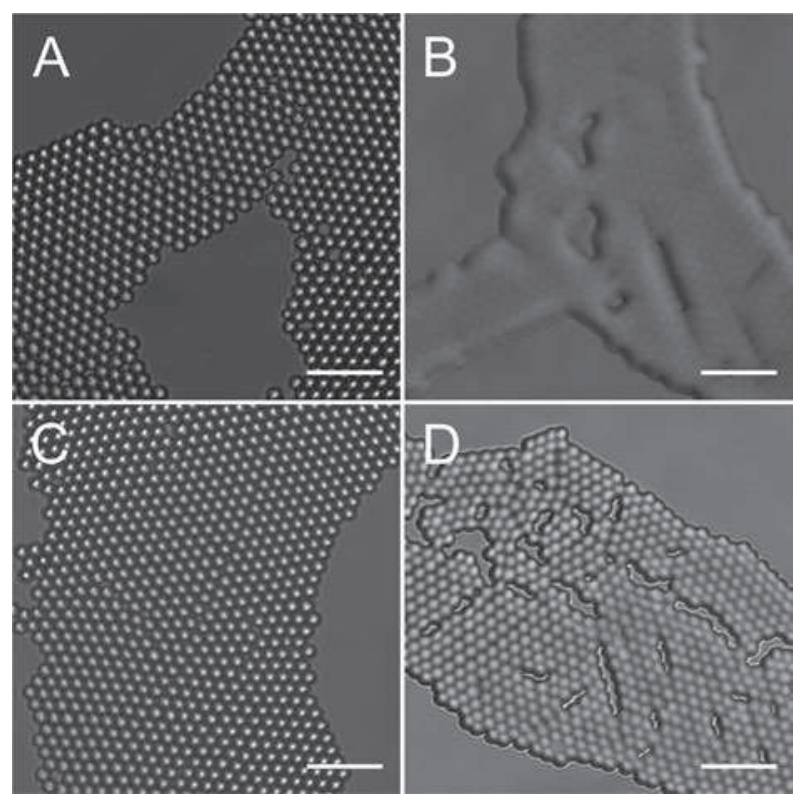

Fig. 2 Temperature stability of non crosslinked and crosslinked PMMA particles. Particle layers of non crosslinked (A) and crosslinked particles (C) were deposited on microscopy coverslips. Both samples were tempered for $1 \mathrm{~h}$ at $200{ }^{\circ} \mathrm{C}$. Micrograph B shows the completely coalesced particle layer after tempering, whereas the particle layer composed of previously crosslinked colloids, illustrated in $D$, shows minor signs of melting after the same heating time. Scale bars: $10 \mu \mathrm{m}$. content of non-crosslinked PMMA. Nevertheless the colloidal crystallites consisting of photo-crosslinked PMMA colloids showed a strongly increased thermal stability and structural integrity which can be attributed to the high degree of photocrosslinking in the illuminated particles as shown by the FT-IR, UV/VIS and gelation studies mentioned above.

\section{Preparation of formstable non-spherical PMMA microparticles}

As a first example for the engineering of this new particle system, we applied the previously described thermo-mechanical stretching procedure to the photo-crosslinkable colloids which have a diameter of $1.6 \mu \mathrm{m}$ (LPX02). We chose these particles because of their intermediate diameter, which facilitates optical microscopy on the resulting ellipsoids in order to study changes in morphology under varying conditions. The stretching procedure can also be applied to much smaller PMMA particles with diameters down to at least $\sim 300 \mathrm{~nm} .{ }^{30}$ Before stretching, we determined the glass transition temperature of the respective particles by differential scanning calorimetry (DSC). The glass transition temperature is located at $110{ }^{\circ} \mathrm{C}$ which is comparable to the $T_{\mathrm{g}}$ of PMMA (Fig. S4 $\uparrow$ ). After embedding in a PDMS matrix, the photo-crosslinkable PMMA particles were thermo-mechanically stretched by a factor of 2.3. As with non-crosslinkable PMMA particles, this yields well-defined ellipsoids.

After isolation by film degradation and redispersion in decalin as described in the Experimental section, $70 \mathrm{mg}$ of the elliptical microparticles dispersed in $2 \mathrm{ml}$ of decalin were illuminated in dispersion for $10 \mathrm{~h}$ to guarantee sufficient photo-crosslinking. In order to study their thermal stability the ellipsoids were thus subjected to the conditions established for the procedure used to covalently lock the steric stabilizer on the particle surface. The latter is performed in order to prevent detachment of the stabilizer in certain dispersants. In detail, after adding $7.9 \mathrm{mg}$ of the ring-opening catalyst $N, N$-dimethyldodecylamine (Armen DMCD; Sigma Aldrich) to the ellipsoids dispersed in decalin, the mixture composed of $5 \mathrm{ml}$ dispersion, containing $70 \mathrm{mg}$ of particles, is stirred at $130{ }^{\circ} \mathrm{C}$ for $6 \mathrm{~h}$ in order to generate ester linkages between the particle surface and the stabilizer backbone. This procedure cannot be applied to ellipsoids prepared from standard PMMA particles, because these quickly relax towards the spherical shape at the elevated temperatures necessary (Scheme 1). As illustrated in Fig. 3, photo-crosslinked ellipsoids almost completely maintained their initial shape and aspect ratio during this procedure, whereas the non-crosslinked particles, which are comparable to common PMMA ellipsoids, considerably relaxed already during the first hour. The change of particle morphology can easily be recognized by optical microscopy. According to the optical micrographs, the shortening of the illuminated particles is finished after the first hour of heating and the shape of the colloids was maintained during the remaining heating time.

Minor changes in particle shape and morphology during stabilizer locking have been quantified using transmission electron microscopy (TEM) (inset of Fig. 3). Samples were prepared by diluting the tempered dispersions $1: 1000$ with 

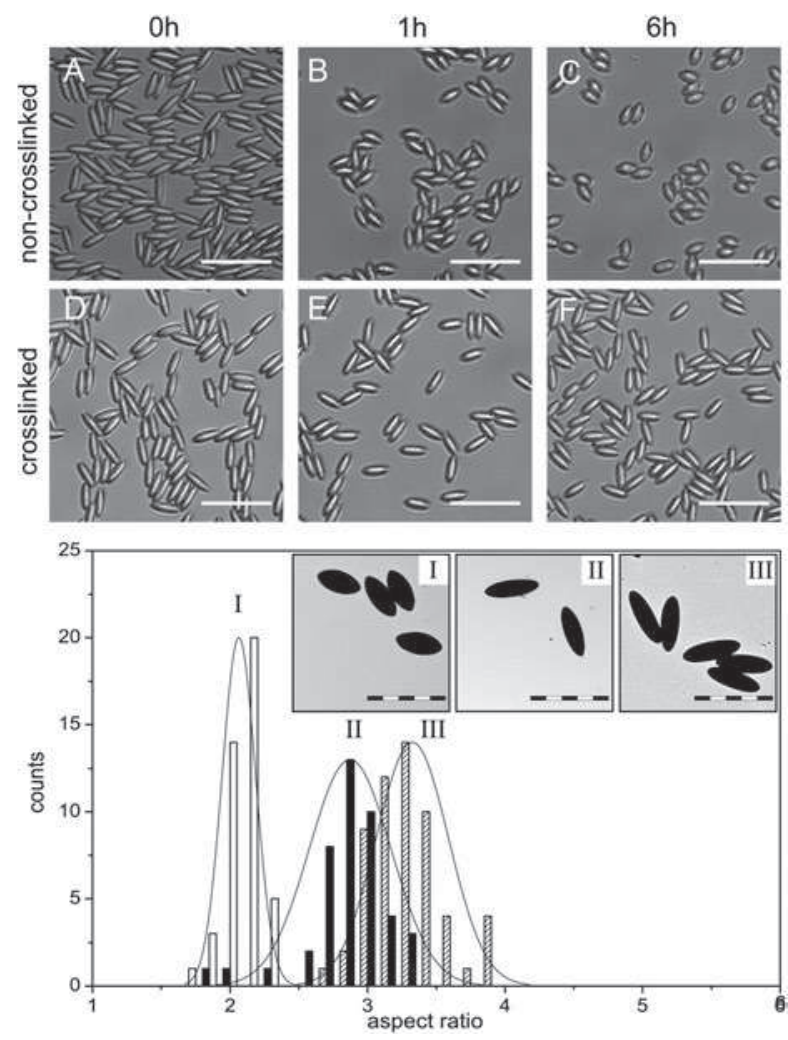

Fig. 3 Relaxation behavior of ellipsoids in dispersion at elevated temperatures. The optical micrographs show ellipsoids spread on microscopy coverslips after stirring them for different times at $130{ }^{\circ} \mathrm{C}$ in decalin. The upper row (A C) illus trates the relaxation behavior of the non crosslinked ellipsoids. The bottom row (D F) shows micrographs of the photo crosslinked counterparts heated for the same time, given on the top of each column. The bottom part of the figure displays the aspect ratio distributions of photo crosslinkable ellipsoids extracted from TEM images. The shaded distribution (III) was obtained by analyzing a population comprising 57 ellipsoids freshly dispersed after the stretching proce dure. The black distribution (II) is derived from 43 crosslinked ellipsoids after $6 \mathrm{~h}$ of tempering and the white distribution (I) represents the same number of non crosslinked ellipsoids after the same heating time. Representative TEM images used to extract the aspect ratios of the corresponding particle sample are given in the inset. All distributions were approximated with a Gaussian distribution highlighting both the respective mean value and FWHM. The scale bars of the inset images and the DIC micrographs correspond to $5 \mu \mathrm{m}$ and $10 \mu \mathrm{m}$ respectively.

decalin such that at most 10 particles could be found in each sector $(50 \mu \mathrm{m} \times 50 \mu \mathrm{m})$ of the TEM grid after drying. This deposition protocol ensures that the analyzed particles are lying flat on the substrate on a plane perpendicular to the optical axis of the TEM microscope.

For the analysis, we mainly focused on the aspect ratio, defined as the ratio of particle length to width. For each sample, we analyzed the dimensions of 43 to 57 particles. The respective aspect ratio distributions of photo-crosslinked and non-crosslinked ellipsoids before and after tempering during $6 \mathrm{~h}$ are illustrated in Fig. 3. They are fitted with a Gaussian distribution function indicating both the mean value and the full width at half maximum (FWHM) of each particle population. This analysis indicates that the non-crosslinked ellipsoids relaxed by $40 \%$ to an aspect ratio of 2.0 during $6 \mathrm{~h}$. By contrast, their photo-crosslinked pendants only slightly relaxed by $12 \%$ to a final aspect ratio of 2.9. In the latter case, the form relaxation is attributed to a certain flexibility of the polymer meshes of the microgel network. Besides the reduction in the aspect ratio, the FWHM decreased significantly for the non-illuminated particles, but their relative polydispersity, defined as the ratio between the FWHM and the mean value, remained almost constant.

The inset of Fig. 3 illustrates exemplary TEM micrographs of the respective particle sample used for statistical analysis. These results underline the formstability of the illuminated particles at high temperatures compared to standard PMMA ellipsoids. We observed the same difference concerning the thermal stability of dry ellipsoids spread out on a microscopy coverslip. Isolated non-crosslinked particles exhibit melted regions (Fig. S7†). By contrast, the photo-crosslinked ellipsoids only showed the same reduction in length as observed during heating in dispersion. Apart from this, only minor signs of melting on the particle surface compared to the results described for the spherical particles (Fig. 2d) were observable. Following the stabilizer locking procedure, the particles could be successfully redispersed in decalin via sonication, even after several centrifugation and washing steps with acetone.

The long-term stability of the obtained dispersion shows that the stabilizer was successfully locked on the particle surface. In order to demonstrate the unique solvent stability of the photocrosslinked elliptical particles, the final particle samples were dispersed in acetone as depicted in the micrographs of Fig. 4A. Under these conditions, standard PMMA ellipsoids immediately
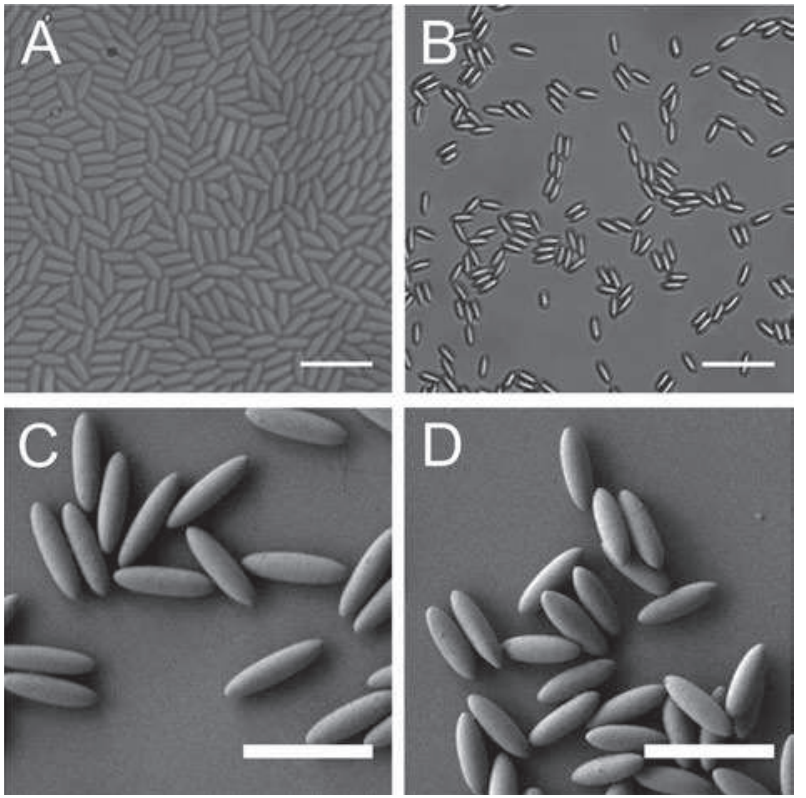

Fig. 4 Solvent stability and swelling of photo crosslinked ellipsoids. Differential interference micrographs of photo crosslinked PMMA ellipsoids dispersed in acetone settled on the bottom of the sample chamber, before (A) and after (B) evaporation of the acetone. SEM images of photo crosslinkable ellipsoids before (C) and after (D) illumination and repeated washing with acetone and storage therein for several weeks. Scale bars of optical micrographs and SEM images correspond to $10 \mu \mathrm{m}$ and $5 \mu \mathrm{m}$ respectively. 
dissolve. Photo-crosslinked ellipsoids, by contrast, keep their morphological integrity except for reversible swelling by a factor of $\sim 3.5$ by volume. Even in the solvent swollen state the particles almost maintain their initial aspect ratio. After deposition and drying of the ellipsoids from acetone, only minor changes in particle shape were observable using optical microscopy (Fig. 4B). In the following, we checked by SEM whether the acetone treatment leads to surface defects of the ellipsoids. Such dissolution defects could be caused by insufficient crosslinking leading to local polymer dissolution on the particle surface. SEM images revealed no defects on the particle surface. Also the SEM data indicate only a minor decrease in aspect ratio compared to their initial shape (Fig. 4C) with the same magnitude as already observed for the previously reported thermal relaxation experiments (Fig. 4D). These results demonstrate that the crosslink density is homogeneously distributed throughout the whole volume of the ellipsoid, corroborating both the statistical distribution of the photo-crosslinkable comonomer and the homogeneity of the photo-crosslinking process.

\section{Photolithographic masking}

The usage as precursor particles to generate shape stable microgel particles is only one possible application of the photocrosslinkable PMMA particles. In order to demonstrate their versatility, we point out that these particles might also be of

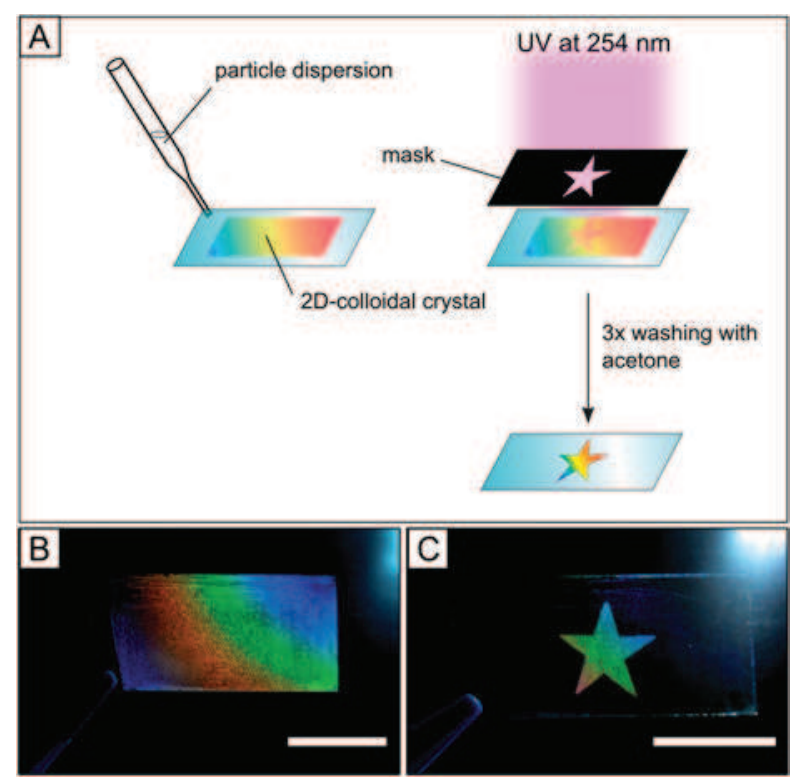

Fig. 5 Photolithography with crosslinkable PMMA particles. Schematic illustration of the photolithographic masking procedure (A). The particle dispersion is deposited on a microscopy coverslip and spread over the surface followed by the immediate formation of colloidal crystalline particle layers during dispersant evaporation (rainbow coloured area). After depositing the paper mask on the sample, the assembly is illuminated with a lab UV lamp at $254 \mathrm{~nm}$ for $30 \mathrm{~min}$. Subsequent washing with acetone leads to selective dissolution of non illuminated areas, whereas illuminated, and hence crosslinked, parts remain intact. Colloidal crystalline coating composed of photo crosslinkable particles with a mean diameter of $1.15 \mu \mathrm{m}$ (LPX01) on a microscopy coverslip after illumination through the paper mask, before (B) and after repeated washing with acetone (C). For the photographs, the samples were backlit with an LED flashlight. Scale bars: $2 \mathrm{~cm}$. interest for photolithographic applications. Due to the pronounced difference in the thermal stability and solvent resistance of photo-crosslinked and non-crosslinked microparticles combined with their tendency to organize in colloidal crystalline areas, these particles can be used for photolithographic patterning of colloidal crystalline particle layers, as shown in Fig. 5. The photolithographic procedure is schematically illustrated in Fig. 5A. After generating colloidal crystalline monolayers on a microscopy coverslip the sample was illuminated with a UV lamp at $254 \mathrm{~nm}$ through a paper mask for 30 min. The photograph shown in Fig. 5B displays a colloidal crystalline particle layer on a microscope coverslip right after illumination. The particles were solely adsorbed on the glass surface. Therefore, to avoid particle detachment, illuminated samples were developed by rinsing them with acetone. Sonication during acetone treatment caused severe particle detachment even in illuminated areas. Washing the substrates with acetone leads to the selective dissolution and complete removal of non-crosslinked particles, whereas the photo-crosslinked colloidal crystalline areas remain adsorbed on the coverslip and still show strong Bragg diffraction due to the colloidal crystallinity (Fig. 5C). If we intentionally detach the photo-crosslinked particles by sonication, we only observe single particles and no clusters. Therefore we can rule out inter-particle crosslinking during illumination. Despite the comparatively low power of the used UV lamp $(40 \mathrm{~W})$ and the illumination at $254 \mathrm{~nm}$ hitting the short-wavelength edge of the cinnamoyl absorption (Fig. S5 $\dagger$ ), we could still obtain sufficient crosslinking contrast for photolithographic patterning.

\section{Photo-crosslinked PMMA particles and loading with functional compounds}

As mentioned in the introduction, the sterically stabilized, photo-crosslinked and elliptical PMMA particles described above are attractive model systems for investigations of colloidal dispersions. An important prerequisite for the application of the ellipsoids in confocal fluorescence microscopy is the possibility of fluorescent staining. We found two different possibilities to prepare fluorescent formstable ellipsoids. One way is the covalent incorporation of a fluorescent comonomer, which is a well established procedure to generate fluorescently stained colloids. ${ }^{49,50}$ For the labeling procedure, we have selected the fluorescent comonomer Rhodamine B-vinyl benzylester and added this reagent at micromolar concentrations to the polymerization mixture used to prepare the intermediate sized photo-crosslinkable particles LPX02. Following the dispersion polymerization and the subsequent washing steps, the covalently stained particles were photo-crosslinked. After 3 $\mathrm{h}$ of illumination, photobleaching of the fluorescent comonomer was still negligible and the resulting microgel colloids showed strong fluorescence while being dispersed in acetone (Fig. 6B).

The covalent linkage of the fluorophore to the polymer network prevents leakage of the fluorophore even in the solvent swollen state of the photo-crosslinked colloids. Comparison between Fig. 6A and 6B shows the pronounced volume change 


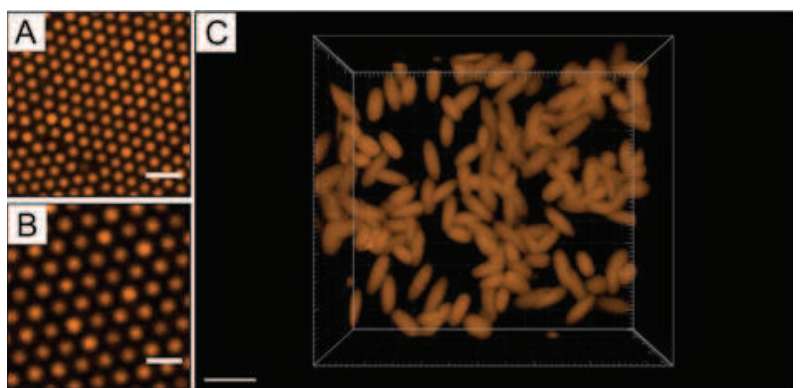

Fig. 6 Fluorescence labeling of crosslinked PMMA particles. A and B are confocal fluorescence micrographs of colloidal crystalline monolayers composed of photo crosslinked covalently stained colloids in decalin (A) and in the solvent swollen state in acetone (B). Particle swelling causes lattice expansion of the colloidal crystalline area. (C) Confocal volume scan of cross linked, elliptical PMMA particles dispersed in decalin. In this case the ellipsoids were fluorescently stained with the swelling/deswelling procedure. Fluorescence was excited at $514 \mathrm{~nm}$ and emission was collected between $550 \mathrm{~nm}$ and $600 \mathrm{~nm}$. Scale bars: $4 \mu \mathrm{m}$.

between the particles dispersed in either decalin (Fig. 6A) or acetone (Fig. 6B). This leads to the larger inter-particle distances in Fig. 6B compared to Fig. 6A.

A second way to stain the particles is to exploit their microgel character. It allows the loading of the photo-crosslinked ellipsoids with cationic fluorescent dyes like Rhodamine B chloride by the well established swelling/deswelling procedure. ${ }^{13}$ This method cannot be applied using common PMMA ellipsoids, since these tend to relax or partially dissolve under these conditions, because the polymer chain mobility in the particles is increased due to the solvent absorption which is crucial for dye penetration into the particles. By contrast, we were able to stain the photo-crosslinked ellipsoids with the fluorescent dye Rhodamine B by adding a solution of the corresponding chloride dissolved in an acetone/cyclohexanone mixture to the particle dispersion in dodecane (see ESI $\dagger$ for details). The gradual addition of the solvent mixture leads to particle swelling, which allows the diffusion of the dye molecules into the particles. After the subsequent deswelling by the addition of decalin, the dye molecules are physically entrapped inside the ellipsoids. Subsequently, the resulting fluorescently stained ellipsoids can be dispersed in non-solvents for PMMA without leaking of the encapsulated dye. Fig. 5C shows a confocal volume scan of the fluorescently stained photo-crosslinked ellipsoids dispersed in decalin on the bottom of a sample chamber. Note that due to the random orientation of the different ellipsoids in the sample some ellipsoids appear spherical depending on the angle of view.

We emphasize that this swelling/deswelling procedure allows the introduction of any species having a solvent in common with PMMA. Loading ellipsoids with a fluorophore is but one example.

\section{Conclusions}

In conclusion, we describe a very versatile new polymer particle system in which the particle synthesis and the particle engineering step are decoupled. The chemical and thermo-mechanical properties of the polymer particles are determined in a photo-crosslinking step after the particle preparation. The photo-crosslinkable PMMA precursor particles were prepared by copolymerizing the photo-crosslinking comonomer CEA with MMA and MA. The particles prepared with our procedure exhibit very low polydispersities, similar to the lowest polydispersities realistically achievable with other preparation techniques.

A broad range of new applications can be realized with these particles. Their unique features are demonstrated in three examples. UV illumination of particles allows their locking in anisotropic shapes after thermo-mechanical deformation. Due to their high degree of crosslinking after the non-invasive photocrosslinking procedure, these anisotropic particles show supreme chemical and thermal stability. This allows their further chemical treatment such as covalent binding of steric stabilizers or subsequent fluorescent labelling by particle swelling.

We have shown how patterned illumination of crosslinkable particle layers affords photolithographic applications. Finally, we have demonstrated the loading of anisotropic, photo-crosslinked particles with functional compounds by swelling and deswelling. We are here by no means limited to using fluorophores. One may endow the particles with magnetic properties by loading them with magnetic composites. Work in this direction is in progress. As one last inspiring feature we mention that the photo-crosslinking may also be undone, viz. by illumination at wavelengths below $260 \mathrm{~nm} .{ }^{42,51}$

\section{Acknowledgements}

We thank Marina Krumova for TEM imaging, Lars Bolk for GPC and DSC measurements, and Stefan Mecking for critical discussions. Funding by the Center for Mesoscopic Structures of the University of Konstanz is gratefully acknowledged. P.P. was associated with the Zukunftskolleg of the University of Konstanz, and gladly thanks Ali Mohraz for his many helpful suggestions.

\section{Notes and references}

1 P. N. Pusey and W. van Megen, Phys. Rev. Lett., 1987, 59, 2083-2086.

2 E. R. Weeks, J. C. Crocker, A. C. Levitt, A. Schofield and D. A. Weitz, Science, 2000, 287, 627-631.

3 A. Yethiraj and A. van Blaaderen, Nature, 2003, 421, 513-517. 4 P. N. Pusey and W. van Megen, Nature, 1986, 320, 340-342.

5 Z. Y. Luo, C. X. Zou, S. Syed, L. A. Syarbaini and G. F. Chen, Colloid Polym. Sci., 2012, 290, 141-150.

6 J. H. Zhang, Z. Q. Sun and B. Yang, Curr. Opin. Colloid Interface Sci., 2009, 14, 103-114.

7 W. Cheng, J. J. Wang, U. Jonas, G. Fytas and N. Stefanou, Nat. Mater., 2006, 5, 830-836.

8 R. P. A. Dullens, E. M. Claesson and W. K. Kegel, Langmuir, 2004, 20, 658-664.

9 D. W. J. Osmond and H. H. Thompson, Great Britain Pat., GB893429, 1962. 
10 L. Antl, J. W. Goodwin, R. D. Hill, R. H. Ottewill, S. M. Owens, S. Papworth and J. A. Waters, Colloids Surf., 1986, 17, 67-78.

11 K. E. J. Barrett and H. R. Thomas, J. Polym. Sci., Part A: Polym. Chem., 1969, 7, 2621-2650.

12 M. T. Elsesser and A. D. Hollingsworth, Langmuir, 2010, 26, 17989-17996.

13 A. D. Dinsmore, E. R. Weeks, V. Prasad, A. C. Levitt and D. A. Weitz, Appl. Opt., 2001, 40, 4152-4159.

14 R. P. A. Dullens, Soft Matter, 2006, 2, 805-810.

$15 \mathrm{H}$. Hu and R. G. Larson, Langmuir, 2004, 20, 7436-7443.

16 V. Prasad, D. Semwogerere and E. R. Weeks, J. Phys.: Condens. Matter, 2007, 19, 113102.

17 A. Mohraz and M. J. Solomon, Langmuir, 2005, 21, 52985306.

18 Y. B. Li, Y. N. He, X. L. Tong and X. G. Wang, J. Am. Chem. Soc., 2005, 127, 2402-2403.

19 M. Seo, Z. H. Nie, S. Q. Xu, M. Mok, P. C. Lewis, R. Graham and E. Kumacheva, Langmuir, 2005, 21, 11614-11622.

20 S. C. Glotzer and M. J. Solomon, Nat. Mater., 2007, 6, 557562.

21 K. Friedemann, T. Corrales, M. Kappl, K. Landfester and D. Crespy, Small, 2012, 8, 144-153.

22 A. P. Cohen, E. Janai, D. C. Rapaport, A. B. Schofield and E. Sloutskin, J. Chem. Phys., 2012, 137, 184505.

23 A. P. Cohen, E. Janai, E. Mogilko, A. B. Schofield and E. Sloutskin, Phys. Rev. Lett., 2011, 107, 238301.

24 M. Mittal and E. M. Furst, Adv. Funct. Mater., 2009, 19, 32713278.

25 P. E. Hopkins, M. Mittal, L. M. Phinney, A. M. Grillet and E. M. Furst, Appl. Phys. Lett., 2011, 99, 133106.

26 D. Schneider, P. J. Beltramo, M. Mattarelli, P. Pfleiderer, J. Vermant, D. Crespy, M. Montagna, E. M. Furst and G. Fytas, Soft Matter, 2013, 9129-9136.

27 J. J. Crassous, H. Dietsch, P. Pfleiderer, V. Malik, A. Diaz, L. A. Hirshi, M. Drechsler and P. Schurtenberger, Soft Matter, 2012, 8, 3538-3548.

28 C. C. Ho, A. Keller, J. A. Odell and R. H. Ottewill, Polym. Int., 1993, 30, 207-211.

29 C. P. Royall, W. C. K. Poon and E. R. Weeks, Soft Matter, 2013, 9, 17-27.
30 Z. K. Zhang, P. Pfleiderer, A. B. Schofield, C. Clasen and J. Vermant, J. Am. Chem. Soc., 2011, 133, 392-395.

31 J. A. Champion, Y. K. Katare and S. Mitragotri, Proc. Natl. Acad. Sci. U. S. A., 2007, 104, 11901-11904.

32 L. C. Lopergolo, A. B. Lugao and L. H. Catalani, Polymer, 2003, 44, 6217-6222.

33 T. Nishikubo, T. Iizawa and M. Hasegawa, J. Polym. Sci., Polym. Lett. Ed., 1981, 19, 113-117.

34 B. J. Kim, Y. Miyamoto, B. W. Ma and J. M. J. Frechet, Adv. Funct. Mater., 2009, 19, 2273-2281.

35 G. T. Carroll, N. J. Turro and J. T. Koberstein, J. Colloid Interface Sci., 2010, 351, 556-560.

36 M. Moritsugu, T. Ogata, S. Kurihara and T. Nonaka, Mol. Cryst. Liq. Cryst., 2005, 443, 117-126.

37 D. Klinger and K. Landfester, Soft Matter, 2011, 7, 1426-1440.

38 G. Kwak, J. U. Choi, K. H. Seo, L. S. Park, S. H. Hyun and W. S. Kim, Chem. Mater., 2007, 19, 2898-2902.

39 V. Enkelmann, G. Wegner, K. Novak and K. B. Wagener, J. Am. Chem. Soc., 1993, 115, 10390-10391.

40 R. C. Nieuwendaal, S. J. Mattler, M. Bertmer and S. E. Hayes, J. Phys. Chem. B, 2011, 115, 5785-5793.

41 Y. Nakamura, J. Chem. Soc., Chem. Commun., 1988, 477-478.

42 A. Lendlein, H. Y. Jiang, O. Junger and R. Langer, Nature, 2005, 434, 879-882.

43 G. Kaupp, Angew. Chem., Int. Ed., 1992, 31, 592-595.

44 I. Fonseca, M. Baias, S. E. Hayes, C. J. Pickard and M. Bertmer, J. Phys. Chem. C, 2012, 116, 12212-12218.

45 P. Gupta, S. R. Trenor, T. E. Long and G. L. Wilkes, Macromolecules, 2004, 37, 9211-9218.

46 M. L. Cao, B. Tong, J. B. Shen, Y. P. Dong and J. G. Zhi, J. Appl. Polym. Sci., 2008, 109, 1189-1196.

47 K. C. Lee and S. Y. Lee, Macromol. Res., 2007, 15, 244-255.

48 I. M. Krieger and F. M. Oneill, J. Am. Chem. Soc., 1968, 90, 3114-3120.

49 G. Bosma, C. Pathmamanoharan, E. H. de Hoog, W. K. Kegel, A. van Blaaderen and H. N. Lekkerkerker, J. Colloid Interface Sci., 2002, 245, 292-300.

50 R. P. A. Dullens, M. Claesson, D. Derks, A. van Blaaderen and W. K. Kegel, Langmuir, 2003, 19, 5963-5966.

51 H. Yang, L. Jia, Z. F. Wang, A. Di-Cicco, D. Levy and P. Keller, Macromolecules, 2011, 44, 159-165. 